\begin{tabular}{||l|l|l|}
\hline \multicolumn{2}{|c|}{ PublisherInfo } \\
\hline \hline PublisherName & $:$ & BioMed Central \\
\hline \hline PublisherLocation & $:$ & London \\
\hline \hline PublisherImprintName & $:$ & BioMed Central \\
\hline \hline
\end{tabular}

\title{
Abstract not submitted for online publication
}

\begin{tabular}{|l|l|l||}
\hline \multicolumn{2}{|l||}{ ArticleInfo } \\
\hline \hline ArticleID & $:$ & 2534 \\
\hline \hline ArticleDOI & $:$ & $10.1186 /$ gb-2010-11-s1-i16 \\
\hline \hline ArticleCitationID & $:$ & I16 \\
\hline \hline ArticleSequenceNumber & $:$ & 16 \\
\hline \hline ArticleCategory & $:$ & Invited speaker presentation \\
\hline \hline ArticleFirstPage & $:$ & 1 \\
\hline \hline ArticleLastPage & $:$ & 1 \\
\hline \hline & & RegistrationDate : 2010-10-11 \\
ArticleHistory & $:$ & OnlineDate \\
\hline \hline ArticleCopyright & $:$ & Schuster; licensee BioMed Central Ltd.2010 \\
\hline \hline & & This article is published under license to BioMed Central Ltd. \\
\hline \hline ArticleGrants & $:$ & \\
\hline \hline ArticleContext & $:$ & 130591111S1S1 \\
\hline \hline
\end{tabular}

Stephan Schuster, ${ }^{\text {Aff1 }}$

Corresponding Affiliation: Aff1

Aff1 The Pennsylvania State University, 310 Wartik Laboratory, University

Park, PA 16802, USA

This PDF file was created after publication. 